\title{
Photodetachment cross-section evaluation using asymptotic considerations
}

\author{
Philippe Babilotte ${ }^{1,2} \cdot$ Mickael Vandevraye ${ }^{1,3}$
}

Received: 20 April 2017 / Accepted: 8 May 2017/Published online: 25 May 2017

(c) The Author(s) 2017. This article is an open access publication

\begin{abstract}
Mathematical calculations are given concerning the evaluation of the negative ions photodetachment crosssection $\sigma$, into a so-called saturation regime. The interaction between a negative ion particle beam and a laser beam is examined under theoretical aspects. A quantitative criterion $S$ is proposed to define the saturation threshold between the linear and the saturated domains, which are both present in this saturation regime. The asymptotic behaviours extracted at the low and high energy limits are used to determine this threshold quantitative criterion $S$ and to evaluate also the photodetachment cross-section $\sigma$. The case of a symmetric gaussian photodetachment laser beam shape is examined according to the proposed formalism, which can be used either for the photo-detachment or photo-ionization processes, and could be potentially used into technological solutions for negative ion neutralisation processes (such as neutral beam injector) in the future fusion energy devices. Estimations onto the errors related to the use of this methodology are given.
\end{abstract}

Experimental and theoritical works performed in: Laboratoire Aimé Cotton, UPR 3321 CNRS, LAC, Université Paris Sud XI, 91405 Orsay Cedex, France

Electronic supplementary material The online version of this article (doi:10.1007/s40094-017-0252-1) contains supplementary material, which is available to authorized users.

Philippe Babilotte

philippe.babilotte@u-bourgogne.fr

1 Laboratoire Aimé Cotton, UPR 3321 CNRS, LAC, Université Paris Sud XI, 91405 Orsay Cedex, France

2 UMR 6303 CNRS, ICB, Université de Bourgogne FrancheComté, UBFC, 21078 Dijon Cedex, France

3 French Ministry of Education, Paris, France
Keywords Other topics in mathematical methods in physics $\cdot$ Metrology $\cdot$ Determination of fundamental constants

\section{Introduction}

Sustainable non-carbon energy systems, renewable energy systems and energy systems that reduce production of waste have been identified as domains of interest in the transition of energy [1]. Hydrogen is the most abundant element in the universe, and can be typically considered as the fuel for any potential fusion reactor. This non-hydrocarbon energy source requires nevertheless to solve many scientific and engineering challenges.

Several dozens of fusion reactors are now scattered around the globe at various stages of completion. The biggest system is the International Thermonuclear Experimental Reactor (ITER). It is being built in France by an international consortium at an estimated cost of $\$ 20$ billion and should be completed by 2027. Other smaller entities work also on the fusion concept, such as General Fusion (Vancouver, Canada), Helion Energy (Redmont, Washington, USA), or Tri Alpha Energy (California, USA) [2].

The nuclear fission involves splitting ${ }^{235} \mathrm{U}$ atoms into smaller atoms, thus releasing a large amount of energy. On the negative side, it requires a finite resource $\left({ }^{235} \mathrm{U}\right)$, expensive (initially and under run-condition) and potentially hazardous power plants (Three Mile Island, Chernobyl, Fukushima accidents), and it produces significant quantities of toxic waste that stay hazardously radioactive for centuries. At the opposite, the fusion has several significant advantages; first, it runs on common standard elements as hydrogen, which is very abundant, non toxic 
and non-fossil. In addition, the fusion reactors cannot melt down, and can be stopped immediately (no possible uncontrollable chain reaction). Lower quantities of radioactive wastes are produced, and fusion could be seen, therefore, as a cleaner power source than the existing ones. Schematically, this technology requires the creation of a plasma to force the atomic nuclei to fuse. This plasma is created under extreme heating and extreme pressure conditions to induce the cloud of free-range electrons and nuclei $\left(T \approx 100 \times 10^{6}{ }^{\circ} \mathrm{C}\right)$. Confinement and control of the plasma stability remain nevertheless points of weakness of the fusion process.

The first method for fusion is the one used by the National Ignition Facility (NIF). The NIF is located in San Francisco (USA, Lawrence National Laboratory, $\$ 5$ billion project cost) and integrates around 192 UV laser beams focused onto a gold cylinder full of a $D+T$ mix, during a $20 \mathrm{~ns}$, inducing a 500 trillion Watt single shot release. It corresponds to a specific Inertial Confinement Fusion (ICF process), as the cylinder (containing $D+T$ ) target simultaneously explodes and implodes.

The second method to get fusion is the most common method, and consists of magnetically controlled plasma, contained in a metallic doughnut, wrapped in electromagnetic coils, which contains and compresses the plasma. In 1957, in the ZETA fusion reactor (UK), tests were conducted for the first time concerning a magnetic field that was used to spatially confine atoms. In 1968, doughnutstructured systems appeared (Tokamak Russian process). Afterwards, in the 1970s, three large-scale tokamaks were developed in Princeton (USA), in Japan, and in the UK. In 2008, the ITER "proof of concept" (and afterwards, the DEMO technological project) $[3,4]$ was started $(300 \mathrm{~m}$ tall, 23,000 tons weight, $10^{5} \mathrm{~km}$ of niobium-tin wire for magnets, $840 \mathrm{~m}^{3}$ expected plasma volume, $\$ 20$ billions cost). Nevertheless, the existence of instabilities in the plasma due to the spiral orbits of particles around the line of electric current (particles in the plasma moving in tight spiral orbits) induced loss of heat, and alternative technological solutions emerged [5-9, 11].

Another (third) method could be, therefore proposed, consisting of creating a single collisional plasma, created using two nose-to-nose cannons ("firing" around $10^{6} \mathrm{~km} \mathrm{~h}^{-1}$ ), firing plasmas straight to each other into a central chamber (collisional regime). Positioned around the central chamber, six Neutral Beam Injector (NBI) introduces $H$ atoms at the edges of the spinning cloud (stabilization keeping the medium hot). In this type of technology, the plasma auto-generates the magnetic field that confines it ("Field Reverse Configuration").

Another (fourth) prototype of fusion reactor is the magnetized target fusion (General Fusion project [2]), in which a spinning vortex of liquid metal is created, and in which plasma is injected into the empty center (squeezing) [5-9].

The future fusion reactor requires therefore to manage a space charge neutralization of negative ions (negative ions $D^{-}$species neutralized into $D^{\circ}$ species, as proposed by CYBELE project [10], Eq. 1), which occurs in the Neutral Beam Injector (NBI) [4] of the fusion reactor [12].

As a first option, negative ion beam neutralization could be realized by injecting a gas into the neutralizer cell, but under a low efficiency level around 55\% (corresponding to an electric efficiency of the heating system limited to $34 \%$ ) [13]. Another possible option is the photoneutralization process $[12,14,15]$ that can be seen as an attractive alternative [16], as no gas is injected into the medium to neutralize the negative ions (less parasitic particles produced, no plasma formation in the cell). The neutralization rate is estimated around 95\%, [17], enhancing in this case the electrical efficiency of the heating system up to $60 \%$ [16].

$D^{-}+h v \rightarrow D^{\circ}+\mathrm{e}^{-}$

Neutralization using photodetachment enhanced by several Fabry-Perot cavities have been also invoked [18]. For all these photoneutralization processes, it requires to have reliable absolute photo-detachment cross-section $\sigma$ values, as this parameter is used afterwards in the different numerical models, for instance, in the Space Charge Models (SCM) [19]. Mathematical models, behaviour laws and physics development in the photodetachment processes $[12,14,20]$ are also greatly required.

Into the present work, benchmark calculations are then given concerning the determination of the absolute crosssection $\sigma$ into a photo-neutralization process, considering the overlap between a laser beam and a negative ion beam. It could be seen as an implementation to the existing mathematical methodology. This photodetachment configuration used into the present work reproduces a gas-free photoneutralization configuration (space charge neutralization), probably involved in the future energy generator demonstrator DEMO [3, 21]. The overlap of the laser sheet and the $D^{-}$negative ion beam takes place outside the nuclear island, in the same configuration that is given in the present work (overlap between the laser and the negative ion outside any ionising radiation conditions).

The absolute photodetachment cross-section $\sigma$ of negative ions quantifies the equivalent surface exposed and available to interact with the photons of the laser beam, corresponding therefore roughly to an interaction probability of the particle with the beam.

The simplest methodology to determine $\sigma$ into photodetachment experiments (2) consists of using a direct calculation (3), introducing the number $N$ of neutral atoms $A$, the number $N_{0}$ of initially illuminated negative ions $A^{-}$, 
and the number of photons per surface unit and per time unit (flux) $\Phi$, for a total duration of illumination $T$, considering the probability $P$ of interaction:

$A^{-}+h v \rightarrow A+\mathrm{e}^{-}$

$P=\sigma \times \Phi \times T \Rightarrow \sigma=\frac{N}{N_{0}} \times(T \times \Phi)^{-1}$

The absolute cross-section $\sigma$ can be also evaluated using a socalled "saturation methodology", which is generally more precise than the direct evaluation methodology [22]. It consists of measuring the evolution of the number $N$ of photoinduced events, changing the light energy $E$ of the laser overlapping the negative ions beam. When this energy is high enough (above the saturation energy $E_{\mathrm{S}}$, that is to say if we consider the case in which $E>E_{\mathrm{S}}$ ), the photodetachment probability [23] or the photo-ionization probability [24, 25] converge on 1 (saturation event, Fig. 1). The pioneering experiments concerning the photodetachment of negative ions, and involving this saturation process are proposed by Hall et al. [26] and by Hotop and Lineberger [27]. This saturation method has been extensively used afterwards $[22,27-30]$. The mathematical model initially developed in 1973 [27] has been afterwards improved by Balling et al. [30]. This saturation methodology has been also used to evaluate the absolute photo-detachment cross-section of trapped ions [31, 32], or into the case of a three-photon set-up [33].

Several mathematical models have been proposed to modelize the saturation effect and to extract $\sigma$ from the measurements, as Cervenan and Isenor [34], considering immobile neutral species interacting with laser beam, or Boulassier [35], considering a gaussian profile interacting homogeneously distributed negative ions, or Blondel et al. [33].

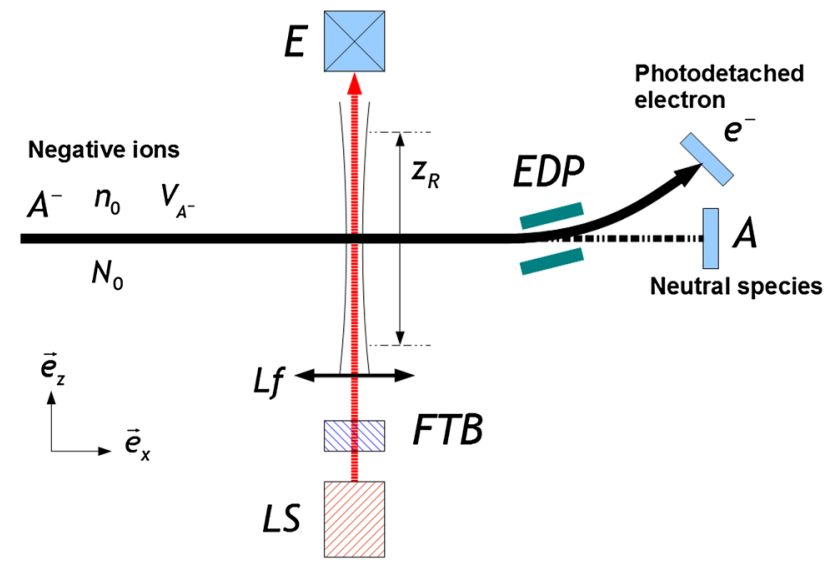

Fig. 1 Typical photodetachment experimental set-up. Basic photoneutralization of negative ions corresponding to an experimental proof. Laser source (LS). Focalisation lenses (Lf). Electrostatic Deviation Plates (EDP). Fluence Tuning Block (FTB). Rayleigh distance $\left(z_{\mathrm{R}}\right)$ of the laser beam. Energy $E$ measured after the interaction of $h v$ with $A^{-}[14]$
The photo-ionisation cross-section can be also determined using this saturation methodology, since the pioneering experiments and estimations proposed by Ambartzumian et al. [24], or Heinzman et al. [25]. Optical transitions can be efficiently characterized using this process [36-41], as well as ions densities and plasma temperature [39, 42]. Nevertheless, this methodology requires a comparison between a reference saturation curve (typically the $O^{-}$reference) and the measured datapoints, motivating to propose new approaches avoiding any comparison processes.

Specifically, in the present work, from two asymptotic considerations, at high and low photodetachment laser irradiation energy $E$, and using the relevant physical hypothesis, a scalar parameter $S$ is introduced as a quantitative criteria characterizing the threshold between unsaturated and saturated regimes present in the photo-excitation process. The expression of the photodetachment cross-section $\sigma$ will be given, without considering any fitting iterative process between a reference curve and the measured datapoints.

In the commonly used experimental configuration (Fig. 1), a single-mode high power (continuous or pulsed) laser beam is used to irradiate a negative ion particle beam, to avoid a possible "bar-code" interaction effect potentially induced by the use of a multimode laser (irregular temporal profile) [14, 27, 43].

The modelized situation considered in the present work consists of two-actors interaction, e.g. between a photon $h v$ and a negative ion particle $A^{-}$. The elementary excitation probability $\mathrm{d} P$ to photodetach one electron from a negative ion $A^{-}$(Fig. 1) during the elementary duration $\mathrm{d} t$ of illumination is given by:

$\mathrm{d} P=\sigma(\lambda) \times \Phi \times \mathrm{d} t$

in which $\sigma(\lambda)$ corresponds to the photodetachment crosssection at the laser irradiation wavelength $\lambda$.

The neutral species density variation $\mathrm{d} n$, found in an elementary volumic element $\mathrm{d} V$ centered onto a point $M$ moving with the negative ions beam is given by:

$\mathrm{d} n=\sigma(\lambda) \times \Phi(M, t) \times\left(n_{0}-n(t)\right) \times \mathrm{d} t$.

We assume for (5) the uniformity of the ion volumic distribution. We assume a constant value for the initial density $n_{0}$ of ions $A^{-}$in the $(y O z)$ plane (Fig. 3).

Considering the two normalized spatio-temporal transverse gaussian laser intensity $f(x, y)$ and $g\left(t-\frac{z}{c}\right)$, which are supposed to be identical along the pulse, we can express the flux $\Phi$ :

$\Phi(M, t)=\Phi(x, y, z, t) \equiv \frac{E}{\hbar \omega} \times f(x, y) \times g\left(t-\frac{z}{c}\right)$.

Two conditions of normalization are used, in accordance with considerations proposed by [30], related to these two distribution functions $f(x, y)$ and $g(t)$ : 


$$
\begin{aligned}
& \int^{x, y} f(x, y) \mathrm{d} x \mathrm{~d} y=1 \\
& \int^{t} g(t) \mathrm{d} t=1 .
\end{aligned}
$$

The flux expression given in (6) allows to rewrite the expression (5) in:

$$
\begin{aligned}
\mathrm{d} n(t)= & \sigma(\lambda) \times \frac{E}{\hbar \omega} \times f(x, y) \times g\left(t-\frac{z}{c}\right) \times\left(n_{0}-n(t)\right) \\
& \times \mathrm{d} t
\end{aligned}
$$

Temporally integrating (9), we find that:

$$
n=n_{0} \times\left(1-\exp \left\{-\sigma(\lambda) \int_{-\infty}^{+\infty} \frac{E}{\hbar \omega} \times f(x, y) \times g\left(t-\frac{z}{c}\right) \mathrm{d} t\right\}\right) .
$$

If $v_{A^{-}}$is the negative ion velocity along the $x$-axis (Fig. 2) and if we consider the modification $x \rightarrow x+v_{A^{-}} t$ and $t^{\prime}=t-\frac{z}{c}$, the density of neutral species produced in a given volume of the negative ion beam, located at $t^{\prime}=0$ in $(x, y, z)$ after its passage inside the laser pulse, can be expressed by:

$$
\begin{aligned}
n= & n_{0} \\
& \times\left(1-\exp \left\{-\sigma(\lambda) \int_{-\infty}^{+\infty} \frac{E}{\hbar \omega} f\left(x+v_{A^{-}} t, y\right) g\left(t^{\prime}\right) \mathrm{d} t^{\prime}\right\}\right) .
\end{aligned}
$$

The ratio $n / n_{0}$ corresponds to a photodetachment probability. The total number $N$ of neutral species photo-produced during the interaction between the laser and the particle beam is afterwards obtained integrating the density onto the whole space, for a given $\lambda$ (single radiation), that is to say considering $\sigma \equiv \sigma(\lambda)$, and considering $t$ as a dummy-variable:

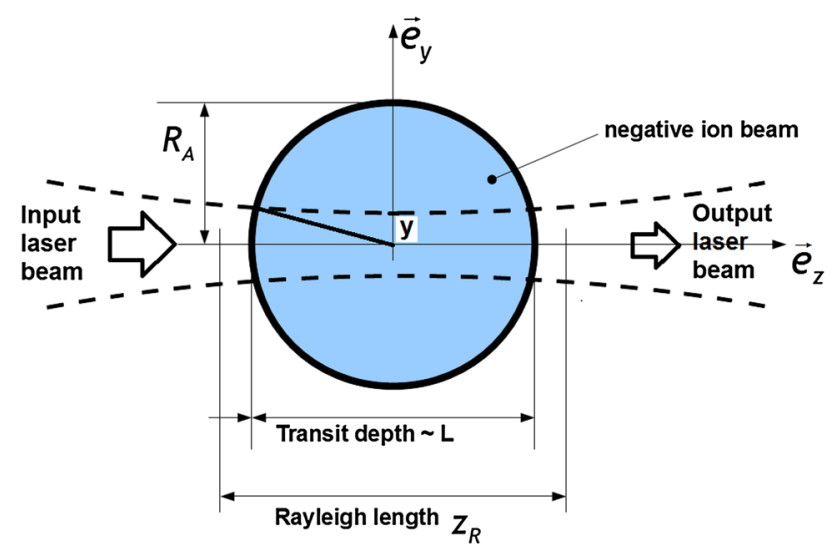

Fig. 2 Lateral view of the interaction area
$N=\int^{x, y, z} n(x, y, z) \mathrm{d} x \mathrm{~d} y \mathrm{~d} z$

It gives therefore:

$$
\begin{aligned}
N=n_{0} L \int_{-\infty}^{+\infty} \int_{-\infty}^{+\infty} \\
\\
\left(1-\exp \left\{-\frac{\sigma E}{\hbar \omega} \int_{-\infty}^{+\infty} f\left(x+v_{A^{-}} t^{\prime}, y\right) g\left(t^{\prime}\right) \mathrm{d} t^{\prime}\right\}\right) \mathrm{d} x \mathrm{~d} y
\end{aligned}
$$

The integration according to the $z$ variable is direct as the Rayleigh area $z_{\mathrm{R}} \approx \frac{\pi w_{0}^{2}}{\lambda}$ (Fig. 2) of the photodetachment laser beam (waist $w_{0}$ ) is supposed much larger than the diameter $L=2 R_{A^{-}}$of the negative ion beam, if $R_{A^{-}}$is the radius of the negative ions beam profile (supposed cylindrical), as we have $z_{\mathrm{R}} \gg L$.

Consequently, from $\frac{L^{2}}{4}=y^{2}+\left(\frac{L(y)}{2}\right)^{2}$ (Fig. 2), the length $L(y)$ of the negative ion beam in the propagation direction $z$ can be considered constant for any altitude $y(L(y) \approx L$, Fig. 3), and we assume that a direct integration in (11) is possible according to the $z$ coordinate.

This approximation concerning the geometry of the interaction area (Fig. 2) is true for the usual photodetachment experiments performed [14], considering a typical waist $w_{0} \approx 70 \mu \mathrm{m}, L \equiv 2 R_{A^{-}} \approx 2 \mathrm{~mm}$ (typical case of a $\approx 1 \mathrm{nA} H^{-}$ion beam), for a typical laser beam at $1064 \mathrm{~nm}$ for instance $(20 \mathrm{~Hz}$, laser pulse width $\tau \approx 30 \mathrm{~ns})$, inducing $z_{\mathrm{R}} \approx 14 \mathrm{~mm} \gg L \approx 2 \mathrm{~mm}$, and considering no particular diffraction phenomenum of the laser beam.

In this direct integration consideration, using the normalization conditions (7) and (8), a direct proportionality

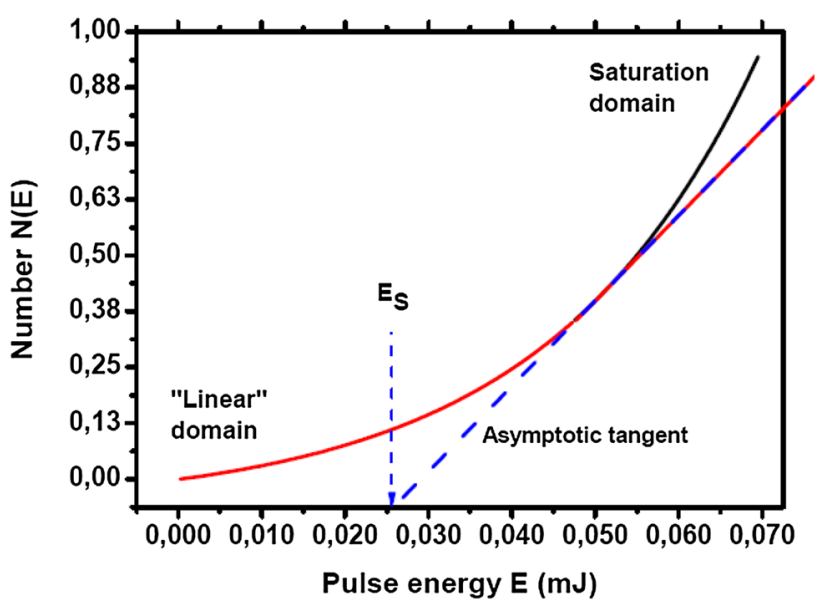

Fig. 3 Typical saturation curve and interception value $E_{\mathrm{S}}$. Typical data for $H^{-}: \lambda=1064 \mathrm{~nm}, v=479,210 \mathrm{~km} \mathrm{~h}^{-1}$ (typical $H^{-}$particle beam), $w_{0}=80 \mu \mathrm{m}, \tau=20 \mathrm{~ns}$ (typical experimental values) 
behaviour between $N$ and $E$ appears at the low energy limit, using a Taylor expansion (for $E$ variable):

$$
\begin{aligned}
N= & n_{0} L \frac{\sigma E}{\hbar \omega} \times \int_{-\infty}^{+\infty} \int_{-\infty}^{+\infty} \int_{-\infty}^{+\infty} f\left(x+v_{A^{-}} t^{\prime}, y\right) g\left(t^{\prime}\right) \mathrm{d} t^{\prime} \mathrm{d} x \mathrm{~d} y \\
& \rightarrow n_{0} L \frac{\sigma E}{\hbar \omega} .
\end{aligned}
$$

In other words, $\forall E \rightarrow 0$, we have $N \rightarrow p_{1} E$, introducing the following quantity:

$p_{1}=n_{0} \times \frac{L \sigma}{\hbar \omega}$.

At the other energy limit $(E \rightarrow \infty)$, the total population of neutral species photo-produced (Fig. 1) can be expressed:

$N=n_{0} V_{\mathrm{S}}+\alpha-\alpha^{\prime}$.

In this expression, $\alpha$ corresponds to the number of neutral species produced into a collisional thermal regime (independently from any laser-matter interaction). $\alpha^{\prime}$ corresponds to the residual number of negative ions undetached into the saturated volume itself (traducing the non-efficiency of the laser-particle interaction). $V_{\mathrm{S}}$ corresponds to the saturation volume. In a first approximation, we assume $\alpha \ll n_{0} V_{\mathrm{S}}$ and $\alpha^{\prime} \ll n_{0} V_{\mathrm{S}}$, inducing then $N \equiv n_{0} V_{\mathrm{S}}$.

At high energy $(E \rightarrow \infty)$, the saturation starts into the volume $\left(V_{S}\right)$ in which the flux is high. Other zones of the overlapping area, in which the saturation is not effective, stay locally into the linear regime described in (15), but a global convergence to the total saturation is nevertheless considered in this case. $V_{\mathrm{S}}$ is determined by geometrical considerations from the condition $G(x, y) \geq S$, if $S$ corresponds to a scalar number. $G(x, y)$ corresponds to the argument of (10), for an ion located at the $(x, y)$ position. It traduces that above a given value of the argument, the saturation exists.

We introduce then $Q=Q_{t} \times Q_{x y}$, and we consider the two normalization conditions (7) and (8) related to the transverse laser intensity profile given in (6). The negative ion velocity field is considered constant along the $x$ geometrical coordinate (supposed mono-axis velocity field according to $x$ ), and we consider for simplification $v_{A^{-}}=v$ :

$g(t)=\frac{1}{\tau} \times \sqrt{\frac{2}{\pi}} \times \mathrm{e}^{-\frac{2 t^{2}}{\tau^{2}}} \equiv Q_{t} \times \mathrm{e}^{-\frac{t^{2}}{2 \tau^{2}}}$

$f(x, y, t)=\frac{2}{\pi w_{0}^{2}} \times \mathrm{e}^{-\frac{2}{w_{0}^{2}} \times\left\{(x+v t)^{2}+y^{2}\right\}} \equiv Q_{x y} \times \mathrm{e}^{-\frac{2}{w_{0}^{2}}\left\{(x+v t)^{2}+y^{2}\right\}}$.

The condition onto the argument $G(x, y)$ of (10) can be given by the inequality $G(x, y) \geq S$, developed in:

$$
\begin{aligned}
G(x, y) \equiv & -Q \frac{\sigma E}{\hbar \omega} \int_{-\infty}^{+\infty} \exp \left(-\frac{2}{w_{0}^{2}}\left[(x+v t)^{2}+y^{2}\right]\right) \\
& \times \exp \left(-\frac{2 t^{2}}{\tau^{2}}\right) \mathrm{d} t \geq S .
\end{aligned}
$$

Considering the calculations into the Appendix 1, and introducing the non-dimensional parameter $\rho=\frac{v^{2} \tau^{2}}{w_{0}^{2}} \geq 0$, and replacing $Q$ by its value, we obtain from (19):

$$
\begin{aligned}
-G(x, y, t)= & \frac{\sigma E}{\hbar \omega} \times\left(\frac{1}{\pi w_{0}^{2}}\right) \times\left(\frac{1}{\sqrt{1+\rho}}\right) \times \exp \left\{-\frac{2 y^{2}}{w_{0}^{2}}\right\} \\
& \times \exp \left\{-\frac{2 x^{2}}{w_{0}^{2}} \times \frac{1}{1+\rho}\right\} .
\end{aligned}
$$

Above the saturation threshold, from (19) and (20), a geometrical condition (21) can then be extracted, introducing the real function $Y(E)$ and the parameter $\delta^{2}=w_{0}^{2} \times(1+\rho)=w_{0}^{2}+v^{2} \tau^{2}:$

$\frac{2}{\delta^{2}} x^{2}+\frac{2}{w_{0}^{2}} y^{2} \leq \operatorname{Ln}\left(\frac{2 \sigma}{S \pi w_{0} \delta} \times \frac{E}{\hbar \omega}\right) \equiv Y(E)$.

All the $(x, y)$ couples of geometrical points concerned by the saturation regime into the overlapping volume are defined by the surface (21). Introducing the two parameters $a$ and $b$, defined by $a=\delta \times \sqrt{\frac{Y(E)}{2}}$ (along the $x$ axis) and $b=w_{0} \times \sqrt{\frac{Y(E)}{2}}$ (along the $y$ axis), $V_{\mathrm{S}}$ can be therefore expressed in:

$V_{\mathrm{S}}=\pi a b L \equiv \frac{L}{2} w_{0} \pi \delta \times\left(\operatorname{LnE}+\operatorname{Ln}\left(\frac{2 \sigma}{S \pi w_{0} \hbar \omega \delta}\right)\right)$.

$N \equiv n_{0} V_{\mathrm{S}}$ is therefore seen as a function depending from the $L n E$ variable, and (22) can be rewritten under $N(\operatorname{Ln} E)=p_{2} \times \operatorname{LnE}+d$, if:

$p_{2}=n_{0} \frac{\pi}{2} L w_{0} \delta$

$d=n_{0} \frac{\pi}{2} L w_{0} \delta \times \operatorname{Ln}\left(\frac{2 \sigma}{S \pi w_{0} \delta} \times \frac{1}{\hbar \omega}\right)$.

$V_{\mathrm{S}}$ should be positive and real, and the expression given in (22) induces then:

$E \geq E_{\mathrm{S}}=\frac{S \pi w_{0} \delta}{2 \sigma} \times \hbar \omega$.

As a consequence of (23) and (24), (22) can be rewritten in:

$V_{\mathrm{S}}=L \times \frac{\pi}{2} \times w_{0} \delta \times \operatorname{Ln}\left(\frac{E}{E_{\mathrm{S}}}\right)$. 
The ratio $\frac{d}{p_{2}}$ is independent from the initial density $n_{0}$ of negative ion, which is unprecisely known:

$\frac{d}{p_{2}}=\operatorname{Ln}\left(\frac{2 \sigma}{S \pi w_{0} \delta} \times \frac{1}{\hbar \omega}\right)$.

According to (25) and (27), the saturation energy threshold $E_{\mathrm{S}}$ is expressed by:

$L n E_{\mathrm{S}} \equiv\left(-\frac{d}{p_{2}}\right)$.

The $\frac{p_{2}}{p_{1}}$ quantity, obtained from (15) and from (23), permits to define the cross-section $\sigma$ :

$\sigma=\frac{p_{1}}{p_{2}} \times \frac{\pi}{2} \times \hbar \omega \times w_{0} \delta$

It exhibits the possibility to extract the photodetachment cross-section value from the two asymptotic behaviours, characterized by $p_{1}$ (corresponding to $E \rightarrow 0$ ) and $p_{2}$ (corresponding to $E \rightarrow \infty$ ), and to define quantitatively a transition limit between the linear domain and the saturation domain, through the parameter $S$ :

$S=\frac{p_{1}}{p_{2}} \times E_{\mathrm{S}} \Rightarrow \sigma=S \frac{\pi w_{0} \delta}{2} \times \frac{\hbar \omega}{E_{\mathrm{S}}}$.

The mathematical expression for $S$ should nevertheless be specified. From (19), introducing $\rho=\frac{v^{2} \tau^{2}}{w_{0}^{2}}$, integrating only over the temporal term, using $x \rightarrow x^{\prime}=\frac{x}{\sqrt{1+\rho}}$ (symmetrization), introducing the parameter $B=\frac{2 \sigma}{\pi w_{0}^{2}} \times \frac{1}{\sqrt{1+\rho}} \times \frac{E}{\hbar \omega}$, the neutral species total number is then evaluated by:

$$
\begin{aligned}
N= & n_{0} L \times \sqrt{1+\rho} \\
& \times \int_{-\infty}^{+\infty} \int_{-\infty}^{+\infty}\left(1-\exp \left\{-B \times \exp \left\{-2 \frac{x^{\prime 2}+y^{2}}{w_{0}^{2}}\right\}\right\}\right) \mathrm{d} x^{\prime} \mathrm{d} y .
\end{aligned}
$$

At the limit $E \rightarrow \infty$, considering the asymptotic consideration $N \rightarrow n_{0} V_{\mathrm{S}}$ at high energy, replacing $B$ by its value, and considering the detailed calculations given in Appendix 2, we obtain:

$$
\begin{aligned}
N \rightarrow & n_{0} L \times \sqrt{1+\rho} \times \frac{\pi w_{0}^{2}}{2} \\
& \times\left\{+\operatorname{Ln}(E)+\operatorname{Ln}\left(\frac{2 \sigma}{\pi w_{0}^{2}} \times \frac{1}{\sqrt{1+\rho}} \times \frac{1}{\hbar \omega}\right)+\gamma\right\}
\end{aligned}
$$

Using (22) and (23), we find from (32) that:

$$
\begin{aligned}
d= & n_{0} L \times \sqrt{1+\rho} \times \frac{\pi w_{0}^{2}}{2} \\
& \times\left\{\operatorname{Ln}\left(\frac{2 \sigma}{\pi w_{0}^{2}} \times \frac{1}{\sqrt{1+\rho}} \times \frac{1}{\hbar \omega}\right)+\gamma\right\} .
\end{aligned}
$$

At the saturation threshold, the laser energy $E$ equalizes the critical energy $E_{\mathrm{S}}$, and it comes:

$\operatorname{Ln}\left(E_{\mathrm{S}}\right)-\operatorname{Ln}\left(\frac{\pi}{2} \times \frac{w_{0}^{2}}{\sigma} \times \sqrt{1+\rho} \times \hbar \omega\right)+\gamma=0$.

Using (30), the threshold criteria $S$ of saturation is finally given by:

$S=\mathrm{e}^{-\gamma}$.

It can be possible then to quantitatively define the saturation threshold, into a photo-ionisation or a photodetachment process, for a gaussian shape. Therefore, the observation of the saturated regime allows to determine the photodetachment cross-section, avoiding to use a comparison methodology [22, 24-34, 36-42, 44] between two saturation curves.

The Eq. (29) and the reading of $E_{\mathrm{S}}$ (Fig. 3), through the asymptotic $E \rightarrow \infty$ behaviour, allows to a direct evaluation of $\sigma$ [14]. For instance, for the negative ion $H^{-}$, using $\lambda=1064 \mathrm{~nm}, v=479,210 \mathrm{~m} \mathrm{~s}^{-1}{ }^{1}{ }^{1} w_{0}=80 \mu \mathrm{m}, \tau=20 \mathrm{~ns}$, $E_{\mathrm{S}}=0.025 \mathrm{~mJ}$, and $\gamma=0.577215$, the obtained value of $\sigma$ is in accordance with all the previous evaluations for $\mathrm{H}^{-}$ given in the literature [14, 45].

The error onto the final result can be quantified using the Fig. 4. The error onto the $E_{\mathrm{S}}$ value is therefore given by $\Delta \sigma=\sigma \times \frac{\Delta E_{\mathrm{S}}}{E_{\mathrm{S}}}$. In Fig. 4, previous parameters are used, introducing $E_{\mathrm{S}}=0.025 \mathrm{~mJ}$, and estimating the impact of a $\pm 0.5 \mathrm{~mJ}$ error onto the cross-section value (value of $E_{\mathrm{S}}$ varying from $1.5 \times 10^{-5}$ to $3.0 \times 10^{-5}$ onto the abscissa axis, corresponding to this uncertainty). $\pm \Delta \sigma$ is then directly read onto the vertical axis in Fig. 4.

\section{Conclusion}

In the present work, a new approach proposes to measure the photo-excitation cross-section $\sigma$, which is a basic physical quantity independent from any experimental conditions, and characterizing an interaction process between two entities. The cross section is basically linked to the collision probability $P$ per unit time $t$ for an incident flux $\Phi$ of particles to impact a target, and is basically defined using $\frac{\mathrm{d} P}{\mathrm{~d} t}=\sigma \times \Phi$.

The first (old) approach uses directly $\sigma=\frac{N}{N_{0}} \times \frac{1}{T} \times \frac{1}{\Phi}$, requiring the knowledge of the number $N_{0}$ of illuminated atoms (resp. $N$ for the excited atoms), during the total illumination time $T$, for an incident flux $\Phi$. This approach could be unprecise, considering possible underlying nonlinear effects, targets potentially exposed to different illuminations $\Phi$, or considering a time of illumination $T$ that could be different according to the spatial coordinates, or

\footnotetext{
${ }^{1}$ Typical orders of magnitude are found from $5 \times 10^{5} \mathrm{~m} \mathrm{~s}^{-1}$ (accelerated ions) to $1 \mathrm{~km} \mathrm{~s}^{-1}$ (trapped ions) [32].
} 


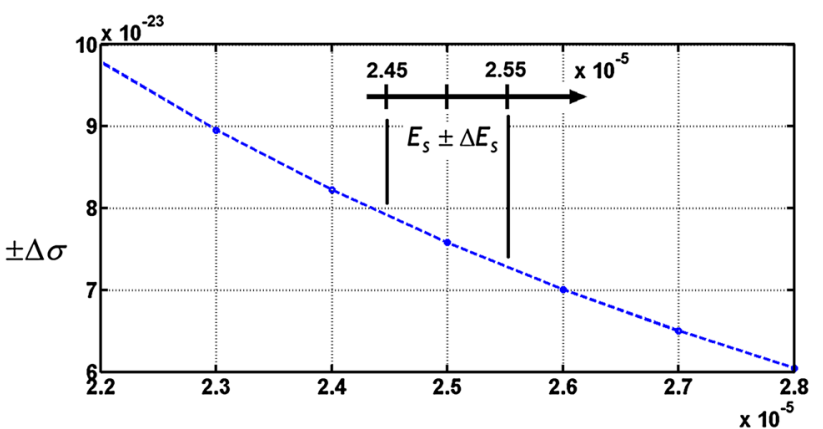

Fig. 4 Numerical estimation of the error induced onto the $\sigma$ final value $( \pm \Delta \sigma)$. Parameters used for the simulation: $w_{0}=80 \mu \mathrm{m}$, $\tau=20 \mathrm{~ns}, \lambda=1064 \mathrm{~nm}, v=479,210 \mathrm{~m} \mathrm{~s}^{-1}, E_{\mathrm{S}}=25 \mu \mathrm{J}$ asymptotically determined (at $\pm \Delta E_{\mathrm{S}}$ ) in Fig. $3, \pm \Delta E_{\mathrm{S}}$ estimated on Fig. 3 at $\pm 0.5 \mu \mathrm{J}$

considering possible errors onto counting efficiencies itself $\left(N_{0}\right.$ and $\left.N\right)$.

The second approach to evaluate $\sigma$ use the criteria $\sigma \times$ $\Phi T=1$ at $63 \%$ for a saturation process defined under $P=1-\exp (-\sigma \Phi T)$. This approach (Fig. 5) does not require to evaluate $N$ and $N_{0}$, but $\Phi$ and $T$ remain to be measured anyway.

The new approach developed into the present work proposes to use the asymptotic behaviours present intrinsically into the saturation process and to develop a calculation based onto the density $n=n_{0} \times\left(1-\exp \left\{-\sigma \int\right.\right.$ $\Phi(t) \mathrm{d} t\})$ of photo-excited ions, considering a laser beam based onto a $f(x, y)$ spatial profile and a $g(t)$ temporal profile, and considering the ion velocity $v$, conducing to $n=n_{0} \times\left(1-\exp \left\{-\sigma \int_{-\infty}^{+\infty} \frac{E}{h \omega} \times f(x+v t, y) \times g(t) \mathrm{d} t\right\}\right)$. Considering a standard Gaussian beam, after volume integration, using $\delta=\sqrt{w_{0}^{2}+v^{2} \tau^{2}}$, it comes the expression of the total number of particles $N$, given by $N=n_{0} \frac{\pi}{2}$ $L w_{0}^{2} \delta \times\left\{\operatorname{Ln} E-\operatorname{Ln}\left(\frac{\pi w_{0} \delta \hbar \omega}{2 \sigma}\right)+\gamma-E i(-B)\right\}$. Asymptotically, this total number of ions converges to $N=n_{0} \frac{\pi}{2} L w_{0}^{2} \delta \times\left\{L n E-\operatorname{Ln}\left(\frac{\pi w_{0} \delta h \omega}{2 \sigma}\right)+\gamma\right\}$. Defining the saturation energy $\operatorname{Ln} E_{\mathrm{S}}=\operatorname{Ln}\left(\frac{\pi w_{0} \delta \hbar \omega}{2 \sigma}\right)+\gamma$, it conduces then to the expression of the cross-section $\sigma=\frac{\pi \mathrm{e}^{-\gamma}}{2} \times \frac{\hbar \omega}{E_{\mathrm{S}}} \times w_{0} \delta$, introducing the two asymptotic parameters $p_{1}$ (for $E \rightarrow 0$ ) and $p_{2}$ (for $E \rightarrow \infty$ ). In the present study, the saturation volume $V_{\mathrm{S}}$ is defined cylindric, exhibiting an elliptic base.

Using a composite profile defined by $\Phi(M, t)=\frac{E}{\hbar \omega} \times g\left(t-\frac{z}{c}\right) \times f(x, y)$, and a Gaussian form of these two (temporal and spatial) profiles $g\left(t-\frac{z}{c}\right)$ and $f($ $x, y)$, it is possible to define a criteria $S$, which corresponds to a quantitative threshold criteria between the saturated regime and the unsaturated regime (linear-saturated transition limit). This is achieved considering the argument of the exponential, through $\frac{\sigma E}{\hbar \omega} \times\left\{\int_{-\infty}^{+\infty} f(x+v t, y) \times g(t)\right.$ $\times \mathrm{d} t\} \geq S$.
Using two temporal and spatial profiles, it comes $\frac{Q \sigma E}{h \omega} \times\left\{\int_{-\infty}^{+\infty} \exp \left\{-\frac{2}{w_{0}^{2}} \times\left((x+v t)^{2}+y^{2}\right)\right\} \times \exp \left\{-\frac{2 t^{2}}{\tau^{2}}\right\} \mathrm{d} t\right\}$ $\geq S$.

It conduces to the intrinsic criteria $S=\mathrm{e}^{-\gamma}$, which quantitatively defines the saturation threshold, if $\gamma$ is the Euler-Mascheroni constant. This proposed new approach has the strong advantage to avoid comparison between laser-matter interaction curves.

Acknowledgements The authors would like to thank Dr. Ch. Blondel and Dr. Cyril Drag for fruitful discussions, and their precious help during experiments onto $H^{-}$, and for overall project guidance.

Open Access This article is distributed under the terms of the Creative Commons Attribution 4.0 International License (http://crea tivecommons.org/licenses/by/4.0/), which permits unrestricted use, distribution, and reproduction in any medium, provided you give appropriate credit to the original author(s) and the source, provide a link to the Creative Commons license, and indicate if changes were made.

\section{Appendix 1}

We simplify the following expression to evaluate the integral present into (19):

$$
\int_{-\infty}^{+\infty}\left\{\exp \left(-\frac{2}{w_{0}^{2}} \times(x+v t)^{2}+y^{2}\right)\right\} \times\left\{\exp \left(-\frac{2 t^{2}}{\tau^{2}}\right)\right\} \times \mathrm{d} t
$$

This infinite integral (36) contains the exponential function of a second order polynomial form, which can be given by $\exp \left\{-a_{2} t^{2}+a_{1}(x) t+a_{0}(x)\right\}$. The three following $a_{0}(x)$, $a_{1}(x)$ and $a_{2}$ expressions can be then considered:

$$
\left\{a_{2}=2 \times\left(\frac{v^{2}}{w_{0}^{2}}+\frac{1}{\tau^{2}}\right) ; a_{1}(x)=-\frac{4 v x}{w_{0}^{2}} ; a_{0}(x)=-\frac{2}{w_{0}^{2}} \times x^{2}\right\}
$$

The following integral identity will be then used, considering $k=\frac{\sigma E Q}{\hbar \omega}$ :

$$
\begin{aligned}
k \times \int_{-\infty}^{+\infty} \exp \left\{-a_{2} t^{2}+a_{1} t+a_{0}\right\} \mathrm{d} t= & k \times \sqrt{\frac{\pi}{a_{2}}} \\
& \times \exp \left(\frac{a_{1}^{2}}{4 a_{2}}+a_{0}\right)
\end{aligned}
$$

\section{Appendix 2}

Due to the symmetry considerations, using a radial function $R$ of $x^{\prime}$ and $y$, defined by $R^{2} w_{0}^{2}=2 \times\left(x^{\prime 2}+y^{\prime 2}\right),(31)$ is changed in: 


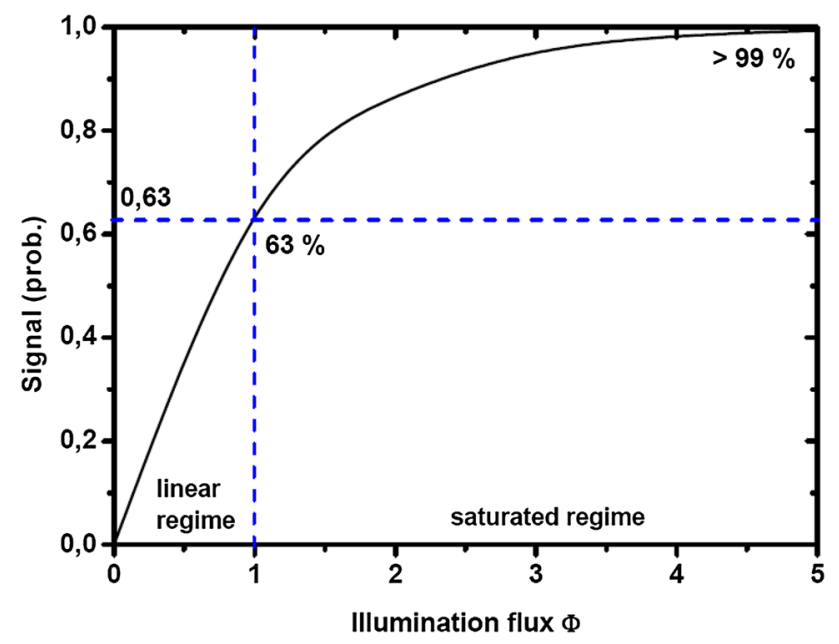

Fig. 5 Saturation criteria defined using the $63 \%$ criteria. The saturation methodology measures the evolution of the number of events varying the fluence of illumination $\Phi$. The evolution of the number of neutral photo-induced neutral species (named "signal") versus the laser pulse energy (flux $\Phi$ ) is given

$$
\begin{aligned}
N= & n_{0} L \times \sqrt{1+\rho} \times \frac{w_{0}^{2}}{2} \times \int_{\theta=0}^{\theta=2 \pi} \int_{R=0}^{+\infty} \\
& \left(1-\exp \left\{-B \times \mathrm{e}^{-R^{2}}\right\}\right) \times R \mathrm{~d} \theta \mathrm{d} R
\end{aligned}
$$

Afterwards, introducing $X=R^{2}, T=\mathrm{e}^{-X}, t=B T$, and integrating (39) according to the $\theta$ coordinate, we have:

$$
\begin{aligned}
N= & n_{0} L \times \sqrt{1+\rho} \times \frac{\pi w_{0}^{2}}{2} \times\left\{\int_{0}^{B}\left(\frac{1-\mathrm{e}^{-t}}{t}\right) \mathrm{d} t\right\} \\
& =n_{0} L \times \sqrt{1+\rho} \times \frac{\pi w_{0}^{2}}{2} \times\{\operatorname{Ein}(B)\}
\end{aligned}
$$

The exponential integral function $\operatorname{Ein}(B)=\int_{0}^{B} \frac{1-\mathrm{e}^{t}}{t} \times \mathrm{d} t$ can be developed using $\operatorname{Ein}( \pm B)=-\operatorname{Ei}(\mp B)+\operatorname{Ln}(B)+\gamma$, introducing the Euler-Mascheroni constant $\gamma$ :

$$
N=n_{0} L \times \sqrt{1+\rho} \times \frac{\pi w_{0}^{2}}{2} \times\{-E i(-B)+\operatorname{Ln} B+\gamma\}
$$

At this step, we modify the $E i(-B)$ expression, considering the following assertion:

$$
\begin{aligned}
E i(-B)= & -\int_{+B}^{+\infty} \frac{\mathrm{e}^{-t}}{t} \mathrm{~d} t=-E i(B) \equiv \\
& -\left(-\int_{-B}^{+\infty} \frac{\mathrm{e}^{-t}}{t} \mathrm{~d} t\right)=\int_{-B}^{+\infty} \frac{\mathrm{e}^{-t}}{t} \mathrm{~d} t
\end{aligned}
$$

As $B>1$, and considering $B \propto E$, we can consider the following inequality between integral behaviours, into the high energy $E$ asymptotic domain:

$$
\int_{-\infty}^{-B} \frac{\mathrm{e}^{t}}{t} \mathrm{~d} t<\int_{-\infty}^{-B} \mathrm{e}^{t} \mathrm{~d} t \equiv \mathrm{e}^{-B}
$$

Therefore, if $E \rightarrow \infty$, we have $\lim _{B \rightarrow \infty}\left\{\mathrm{e}^{-B}\right\}=$ $\lim _{E \rightarrow \infty}\left\{\mathrm{e}^{-E}\right\}=0$, and consequently:

$$
N \rightarrow n_{0} L \times \sqrt{1+\rho} \times \frac{\pi w_{0}^{2}}{2} \times\{+\operatorname{Ln} B+\gamma\}
$$

\section{References}

1. https://ec.europa.eu/energy/en/topics/energy-strategy. Accessed 19 Apr 2016

2. Grossman, L.: A star is born. Time Mag 186(18), 24-33 (2015)

3. Mac Adams, R.: Beyond ITER: neutral beams for a demonstration fusion reactor DEMO. Rev. Sci. Instrum. 85, 02B319 (2014)

4. Simonin, A., Achard, J., Achkasov, K., Bechu, S., Baudouin, C., Baulaigue, O., Blondel, C., Boeuf, J.P., Bresteau, D., Cartry, G., Chaibi, W., Drag, C., de Esch, H.P.L., Fiorucci, D., Fubiani, G., Furno, I., Futtersack, R., Garibaldi, P., Gicquel, A., Grand, C., Guittienne, Ph, Hagelaar, G., Howling, A., Jacquier, R., Kirkpatrick, M.J., Lemoine, D., Lepetit, B., Minea, T., Odic, E., Revel, A., Soliman, B.A., Teste, P.: R\&D around a photoneutralizer-based NBI system (Siphore) in view of a DEMO Tokamak steady state fusion reactor. Nucl. Fusion 55, 12302 (2015)

5. Rostoker, N., Binderbauer, M.W., Monkhorst, H.J.: Colliding beam fusion reactor. Science 278, 1419-1422 (1997)

6. Binderbauer, M.W., TAE team: Dynamic formation of a hot field reversed configuration with improved confinement by supersonic merging of two colliding high-compact toroids. Phys. Rev. Lett. 105, 045003 (2010)

7. Tuszewski, M., TAE team: Field reversed configuration confinement enhancement through edge biasing and neutral beam injection. Phys. Rev. Lett. 108, 255008 (2012)

8. Slough, J., Votroubek, G., Pihl, C.: Creation of a high-temperature plasma through merging and compression of supersonic field reversed configuration plasmoids. Nucl. Fusion 51, 053008 (2011)

9. Laberge, M.J.: An acoustically driven magnetized target fusion reactor. J. Fusion Energy 27, 65-68 (2008)

10. Simonin, A., Garibaldi, P.: Cybele: a large size ion source of modular construction for the Tore-Supra diagnostic injector. Rev. Sci. Instrum. 77, 03A525 (2006)

11. Waldrop, M.: Plasma physics: the fusion upstarts. Nature 511, 398 (2014)

12. Lee, D.H., Brandon, W.D., Pegg, D.J., Hanstorp, D.: Saturated photodetachment for high-efficiency neutral beam injectors. In: IAEA Conference, 25th Fusion Energy Conference, FEC, 46091, $\mathrm{CN}-221$ (2014)

13. Fubiani, G., de Esch, H.P.L., Simonin, A., Hemsworth, R.S.: Modeling of secondary emission processes in the negative ion based electrostatic accelerator of the International Thermonuclear Experimental Reactor. Phys. Rev. Spec. Top. Accel. Beams 11, 014202 (2008) 
14. Vandevraye, M., Babilotte, P., Drag, C., Blondel, C.: Laser measurement of the photodetachment cross section of $\mathrm{H}^{-}$at the wavelength 1064 nm. Phys. Rev. A 90, 013411 (2014)

15. Simonin, A., de Esch, H.P.L., Garibaldi, P., Grand, C., Bechu, S., Bes, A., Lacoste, A.: Ion source development for photoneutralization based NBI system for fusion reactors. In: Fourth International Symposium on Negative Ions, Beams and Sources, NIBS 2014, Conference Proceedings, vol. 1655, pp. 05 0002-1-05 0002-10 (2014)

16. Surrey, E., King, D.B., Lister, J., Porton, M., Timis, W., Ward, D.: In: Symposium on Fusion Technology, Porto (2001)

17. Giruzzi, G., et al.: Final Report for TA WP13-SYS01-B, EFDA Power Plant Physics and Technology. Technical report

18. Fiorucci, D., Chaibi, W., Man, C.N., Simonin, A.: In: Fourth International Symposium on Negative Ions, Beams and Sources, NIBS 2014. Conference Proceedings (2014)

19. Surrey, E., Porton, M.: Space charge neutralization od DEMO relevant negative ion beams at low gas density. In: Second International Symposium on Negative Ions, Beams and Sources, NIBS 2011. Conference Proceedings, vol. 1390, pp. 482-493 (2011)

20. Drag, C., Vandevraye, M., Babilotte, P., Blondel, C.: Laser measurement of the photodetachment cross-section of $\mathrm{H}^{-}$at the wavelength $1064 \mathrm{~nm}$. In: Fourth International Symposium on Negative Ions, Beams and Sources, NIBS 2014. Conference Proceedings, vol. 1655 , p. 57 (2014)

21. Chaibi, W., Blondel, C., Cabaret, L., Delsart, C., Drag, C., Simonin, A.: Photoneutralization of negative ion beam for future fusion reactor. In: Fourth International Symposium on Negative Ions, Beams and Sources, NIBS 2008. Conference Proceedings (2008)

22. Lee, D.H., Brandon, W.D., Pegg, D.J., Hanstorp, D.: Cross section for photodetachment of the excited $\mathrm{C}^{-}(2 \mathrm{D})$ ion using fast ion-laser-beam electron spectroscopy. Phys. Rev. A 56, 1346-1350 (1997)

23. Ghose, J.K., Kumar, B.: Phys. Rev. D 13, 2736 (1965)

24. Ambartzumian, R.V., Furzikov, N.P., Letokhov, V.S., Puretsky, A.A.: Measuring photoionization cross-sections of excited atomic states. Appl. Phys. 9, 335-337 (1976)

25. Heinzmann, U., Schinkowski, D., Zeman, H.D.: Appl. Phys. 12, 113 (1977)

26. Hall, J.L., Robinson, E.J., Branscomb, L.M.: Laser doublequantum photodetachment of $\mathrm{I}^{-}$. Phys. Rev. Lett. 14, 1013-1017 (1965)

27. Hotop, H., Lineberger, W.C.: Dye-laser photodetachment studies of $\mathrm{Au}^{-}, \mathrm{Pt}^{-}, \mathrm{PtN}^{-}$, and $\mathrm{Ag}^{-}$. J. Chem. Phys. 58, 2379-2387 (1973)

28. Dellwo, J., Liu, Y., Tang, C.Y., Pegg, D.J., Alton, G.D.: Photodetachment cross sections for $\mathrm{Li}^{-}$. Phys. Rev. A 46, 3924-3928 (1992)

29. Lee, D.H., Pegg, D.J., Hanstorp, D.: Fast ion-beam photoelectron spectroscopy of $\mathrm{Ca}^{-}$: cross sections and asymmetry parameters. Phys. Rev. A 58, 2121-2127 (1998)
30. Balling, P., Brink, C., Andersen, T., Haugen, H.K.: Absolute photodetachment cross sections of $\mathrm{Cu}^{-}$. J. Phys. B 25, L565L571 (1992)

31. Kwon, N., Armstrong, P.S., Olsson, T., Trainham, R., Larson, D.J.: Single and multiphoton detachment from stored $\mathrm{F}^{-}$ions. Phys. Rev. A 40, 676-682 (1989)

32. Champeau, R.J., Crubellier, A., Marescaux, D., Pavolini, D., Pinard, J.: Photodetachment of trapped negative ions: I. Experimental methods and near-threshold cross sections. J. Phys. B 31, 249-259 (1998)

33. Blondel, C., Champeau, R.J., Crance, M., Crubellier, A., Delsart, C., Marinescu, D.: Measurement of the three-photon detachment cross sections of the negative ions of iodine, bromine and fluorine at the wavelength 1.064 micrometer. J. Phys. B 22, 1335 (1989)

34. Cervenan, M.R., Isenor, N.R.: Multiphoton ionization yield curves for gaussian laser beams. Optim. Commun. 13, 175-178 (1975)

35. Boulassier, J.C.: Photodetachment cross sections for $\mathrm{Li}^{-}$. Nouv. Rev. Opt. 7, 329-340 (1976)

36. He, L.W., Burkhardt, C.E., Ciocca, M., Leventhal, J.J.: Sections for the photoionization of the $6 \mathrm{~s} 6 \mathrm{p} 1 \mathrm{P}$ excited state of barium. Phys. Rev. Lett. 67, 2131-2134 (1991)

37. Shahzada, S., Ijaz, P., Shah, M., Sami-Ul-Haq, Ahmed, M., Nadeem, A.: Photoionization studies from the $3 \mathrm{p} 2 \mathrm{P}$ excited state of neutral lithium. J. Opt. Soc. Am. 29, 3386-3392 (2012)

38. Smith, A.V., Goldsmith, J.E.M., Nitz, D.E., Smith, S.J.: Absolute photoionization cross-section measurements of the excited 4D and 5S states of sodium. Phys. Rev. A 22, 577-581 (1980)

39. Burkhardt, C.E., Libbert, J.L., Xu, J., Leventhal, J.J.: Application to determination of atomic densities: absolute measurement of photoionization cross sections of excited atoms. Phys. Rev. A 38, 5949-5952 (1988)

40. Taillet, J.: Détermination des concentrations en ions négatifs par photodétachement-éclair. C.R. Acad. Sci. Paris Fr. (1969)

41. Bacal, M., Hamilton, G.W.: $\mathrm{H}^{-}$and $\mathrm{D}^{-}$production in plasmas. Phys. Rev. Lett. 42, 1538-1540 (1979)

42. Devynck, P., Auvray, J., Bacal, M., Berlemont, P., Bruneteau, J., Leroy, R., Stern, R.A.: Photodetachment technique for measuring $\mathrm{H}^{-}$velocities in a hydrogen plasma. Rev. Sci. Instrum. 60, 2873-2878 (1989)

43. Siegman, A.E.: Lasers. University Science Books, Mill Valley (1986)

44. Bacal, M.: Photodetachment diagnostics techniques for measuring negative ion densities and temperatures in plasmas. Rev. Sci. Instrum. 71, 3981-4006 (2000)

45. Andersen, T.: Laser measurement of the photodetachment cross section of $\mathrm{H}^{-}$at the wavelength $1064 \mathrm{~nm}$. Phys. Rep. 394, 157-313 (2004) 